\title{
Coilin-dependent snRNP assembly is essential for zebrafish embryogenesis
}

\author{
Magdalena Strzelecka ${ }^{1}$, Simon Trowitzsch $^{2}$, Gert Weber $^{2}$, Reinhard Lührmann ${ }^{2}$, Andrew C Oates ${ }^{1}$ \& Karla M Neugebauer ${ }^{1}$ \\ Spliceosomal small nuclear ribonucleoproteins (snRNPs), comprised of small nuclear RNAs (snRNAs) in complex with snRNP- \\ specific proteins, are essential for pre-mRNA splicing. Coilin is not a snRNP protein but concentrates snRNPs and their assembly \\ intermediates in Cajal bodies (CBs). Here we show that depletion of coilin in zebrafish embryos leads to CB dispersal, deficits \\ in snRNP biogenesis and expression of spliced mRNA, as well as reduced cell proliferation followed by developmental arrest. \\ Notably, injection of purified mature human snRNPs restored mRNA expression and viability. snRNAs were necessary but not \\ sufficient for rescue, showing that only assembled snRNPs can bypass the requirement for coilin. Thus, coilin's essential function \\ in embryos is to promote macromolecular assembly of snRNPs, likely by concentrating snRNP components in CBs to overcome \\ rate-limiting assembly steps.
}

In eukaryotes, organelles delimited by lipid bilayers support the organization of cellular functions, as exemplified by the segregation of chromatin into the cell nucleus. However, many subcellular compartments-nucleoli, Cajal and PML bodies, polar granules, stress granules, $\mathrm{P}$ bodies and others-lack membranes. How these structures contribute to cellular physiology is largely unknown ${ }^{1}$. The Cajal body $(\mathrm{CB})$ is a 0.5 - to $1-\mu \mathrm{m}$ nuclear compartment initially described by Ramon y Cajal in silver-stained sections of vertebrate cerebral cortex ${ }^{2}$. Because the CB is conserved in evolution and is uniquely marked by the protein coilin, it has served as a general model for subnuclear structure and function ${ }^{1,2}$. Numerous factors essential for pre-mRNA splicing, histone mRNA 3'-end processing, telomere maintenance and rRNA processing are concentrated in CBs. Yet CB components exchange rapidly with the nucleoplasm, where most of these processes occur ${ }^{3}$. The challenge, therefore, has been to identify the function of CBs.

Despite the localization of splicing machinery in CBs, they are not the sites of pre-mRNA splicing ${ }^{4,5}$. Splicing occurs throughout the nucleus and is catalyzed by the spliceosome, a macromolecular complex formed on pre-mRNA from five essential snRNPs ${ }^{6}$. Each spliceosomal snRNP comprises a unique 100- to 200-nucleotide snRNA (U1, U2, U4, U5 and U6) that is post-transcriptionally modified and associated with a number of snRNP-specific proteins ${ }^{6,7}$. Several roles for CBs in spliceosomal snRNP biogenesis have been proposed based on the localization of specific steps in snRNP assembly in the CBs of cultured cell lines. After transcription and export to the cytoplasm, U1, U2, U4 and U5 snRNAs receive a heteroheptameric ring of Sm proteins assembled by the SMN complex; subsequently, their $5^{\prime}$ ends are hypermethylated ${ }^{8}$. The Sm ring and trimethylguanosine (TMG) cap are signals for snRNP reimport into the nucleus, where these still-immature core snRNPs first concentrate in $\mathrm{CBs}^{9-12}$. CBs contain scaRNAs, which then guide site-specific modification of the snRNAs ${ }^{13}$. Importantly, intermediates in the final snRNP maturation steps, reflecting RNA structural rearrangements and the recruitment of snRNP-specific proteins, are highly concentrated in $\mathrm{CBs}^{14-17}$. These observations have led to the proposal that CBs are the sites of snRNP assembly, but an essential role for CBs in this process has not been shown.

The coilin protein may hold the key to CB function. Immunoelectron microscopy studies of CBs reveal thread-like, coilin-positive aggregates, suggesting that coilin is integral to $\mathrm{CB}$ structure $^{2}$. Consistent with this, coilin resides longer in CBs than any other examined component in vivo ${ }^{3,18}$. Notably, depletion of coilin results in the dispersal of many CB components. Without coilin, spliceosomal factors become nucleoplasmic, and other classes of CB-localized factors (for example, SMN, fibrillarin and small Cajal body-specific RNAs (scaRNAs)) continue to self-associate in discrete 'residual bodies'19-23. Moreover, tethering of coilin to a particular nuclear site induces formation of a CB at that site ${ }^{24}$. Although it lacks recognizable functional protein motifs, coilin is well conserved in evolution ${ }^{19,21,25}$. Coilin has been shown to multimerize through its N-terminal portion, and interactions with snRNP proteins have been attributed to the $\mathrm{C}$ terminus ${ }^{14,26,27}$. However, coilin has never been identified as a stable component of any of the macromolecular complexes found in CBs, such as the spliceosomal snRNPs, despite exhaustive biochemical study and characterization by $\mathrm{MS}^{4}$. Taken together, this evidence has led to the view that coilin provides the structural scaffold of CBs through self association and a network of low-affinity interactions with CB components ${ }^{1,5,24,28}$.

Coilin clearly has a central role in the integrity of the $\mathrm{CB}$, but the functional consequences to cellular or organismal physiology from

${ }^{1}$ Max Planck Institute of Molecular Cell Biology and Genetics, Dresden, Germany. ${ }^{2}$ Max Planck Institute of Biophysical Chemistry, Göttingen, Germany. Correspondence should be addressed to K.M.N. (neugebau@mpi-cbg.de). 


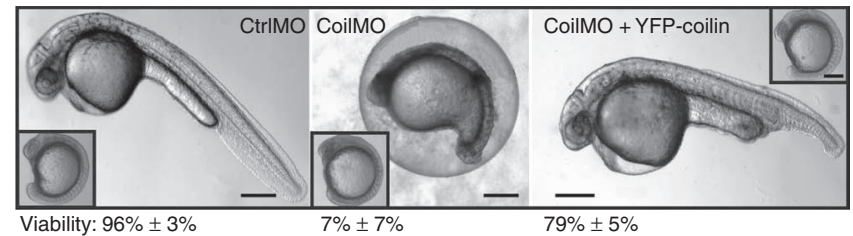

Figure 1 Coilin is essential for embryogenesis. Zebrafish embryos injected with 1 pmol CtrIMO or targeting the $5^{\prime}$ UTR of coilin mRNA (CoilMO) with or without a 0.5 -fmol rescuing dose of YFP-coilin mRNA lacking the CoilMO binding site. Large frames show embryos imaged at 29 h.p.f.; insets, 16 h.p.f. Scale bars, $250 \mu \mathrm{m}$. Values represent average viability in groups of 20-40 embryos \pm s.d. for $n=4$ independent experiments.

a loss of coilin remain unclear. Depletion of coilin from HeLa cells by RNAi slows cell growth ${ }^{20}$, whereas Drosophila coilin-null mutants appear phenotypically wild type ${ }^{21}$. Interestingly, homozygous mouse mutants lacking $85 \%$ of the $\mathrm{C}$-terminal part of the coilin coding region are significantly underrepresented among the progeny of heterozygous parents ${ }^{23,29}$, suggesting that coilin function may become limiting in the context of vertebrate embryogenesis. Here, we take advantage of the rapid, external development of the zebrafish Danio rerio to investigate the function of coilin and CBs. Because of the efficacy of morpholino knockdown in zebrafish, and because the zebrafish embryo is amenable to imaging from fertilization onwards, this model system facilitates analysis of experimental perturbations in a living animal. We have shown that coilin and snRNPs are concentrated in numerous CBs in zebrafish embryos before, during and after activation of the zygotic genome ${ }^{30}$. The present study shows that coilin is required during vertebrate embryogenesis to maintain the supply of assembled spliceosomal snRNPs sufficient for expression of zygotic transcripts.

\section{RESULTS}

\section{Coilin knockdown is embryonic lethal}

To determine the function of coilin in vertebrate embryogenesis, we injected a translation-blocking morpholino (CoilMO) into fertilized zebrafish embryos at the 1-cell stage. Injection of 1 pmol CoilMO led to developmental arrest at the 15- to 16-somite stage ( 16-17 hours post fertilization (h.p.f.)), followed by widespread cell death and embryonic lethality (Fig. 1 and Supplementary Figs. 1 and 2). Arrest was preceded by a systematic decrease in cell proliferation at $\sim 14$ h.p.f., when coilin levels were markedly reduced (Supplementary Figs. 2 and 3 ). Titration experiments revealed that embryonic lethality was dose dependent; embryos injected with less than 1 pmol did not show arrest but a general developmental delay, whereas a higher dose resulted in arrest at the 1- to 3-somite stage ( 10.5-11 h.p.f.) (Supplementary Fig. 1). We designed a second morpholino (CoilMOsplice), targeting the coilin exon 2-intron 2 boundary, to inhibit splicing of coilin pre-mRNA. This 'splicing' morpholino caused developmental arrest at the $\sim 18$ - to 19 -somite stage ( $\sim 18$ h.p.f.); penetrance of the phenotype was lower $(\sim 30 \%)$, likely due to maternally provided coilin $\mathrm{mRNA}^{31}$. We showed specificity of the coilin knockdown by phenotypic rescue with an mRNA encoding yellow fluorescent protein-labeled coilin (YFP-coilin) but lacking the binding site for CoilMO (Fig. 1). We determined rescue in groups of 20-40 embryos injected with 1 pmol of CoilMO with or without YFP-coilin mRNA. In the group injected with CoilMO only, viability at 24 h.p.f. was $7 \% \pm 7 \%$; upon co-injection of YFP-coilin mRNA, viability increased to $79 \% \pm 5 \%$. The YFP-coilin protein was well detected in embryos at 24 h.p.f., consistent with its ability to rescue embryos within this time frame ${ }^{30}$. The majority of the rescued embryos survived for $7 \mathrm{~d}$, at which point we terminated the experiment. We conclude that knockdown of coilin protein in zebrafish embryos compromises cell proliferation and leads to lethality.

Because coilin protein is maternally provided in the embryos of other species, we anticipated that injection of embryos with CoilMO would affect coilin levels progressively, with maternally contributed protein decreasing over time. Immunostaining of CtrlMO-injected embryos with an antiserum specific for zebrafish coilin revealed that nuclear compartments enriched in coilin were present in embryos before and after zygotic genome activation (ZGA) at $\sim 3$ h.p.f., confirming a maternal contribution (Supplementary Fig. 3).
Figure 2 Coilin is essential for Cajal body integrity. (a) Concentration of endogenous snRNP-containing $\mathrm{CBs}$ at $\sim 10$ h.p.f. and dispersal following CoilMO injection is shown by double immunostaining with antibodies against the TMG cap (green) and coilin (red). Single confocal sections are shown. Arrowheads indicate nuclei magnified in the insets $(2.5 x)$. Scale bars, $20 \mu \mathrm{m}$. Pixel intensities in the insets are increased $1.3 \times$ to better visualize the nuclei. (b) YFP-coilin mRNA injection rescues CB morphology. Embryos injected with 1 pmol CoilMO and $0.5 \mathrm{fmol}$ YFP-coilin mRNA (green) immunostained at $\sim 10$ h.p.f. for TMG (red). The negative control is provided in Supplementary Figure 9a. Arrowheads show YFP-coilin and TMGpositive nuclear bodies. Arrows indicate nucleus magnified $2 x$ in the insets. Counts of TMGpositive bodies per nucleus in confocal sections of immunostained preparations revealed that contained $1.7 \pm 0.4$ (mean \pm s.d.) CBs, compared to $0.3 \pm 0.1$ in CoilMO alone and $1.5 \pm 0.3$ in CtrlMO-injected embryos. The values show statistically significant differences from CoilMO alone ( $P=0.005$ for YFP-coilin-injected coilin morphants and $P<0.005$ for CtrIMO). Differences between YFP-coilin-injected coilin morphants and CtrlMO-injected embryos were not significant, according to the two-sample, two-tailed $t$-test ( $n=4$ independent experiments).
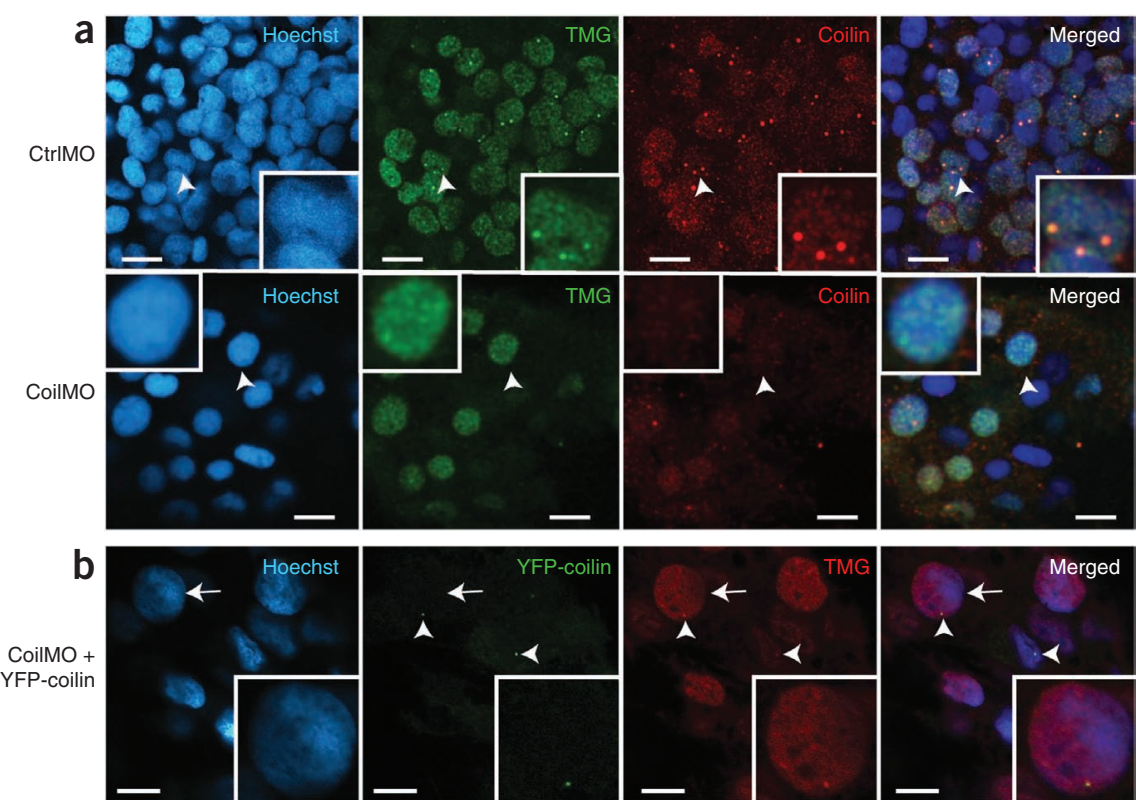

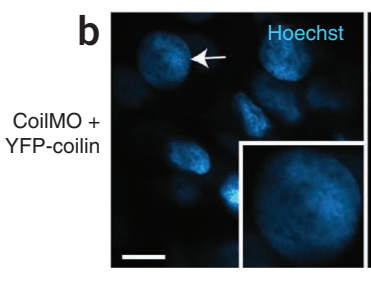


a
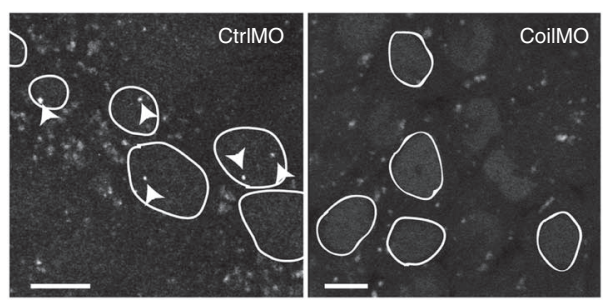

b

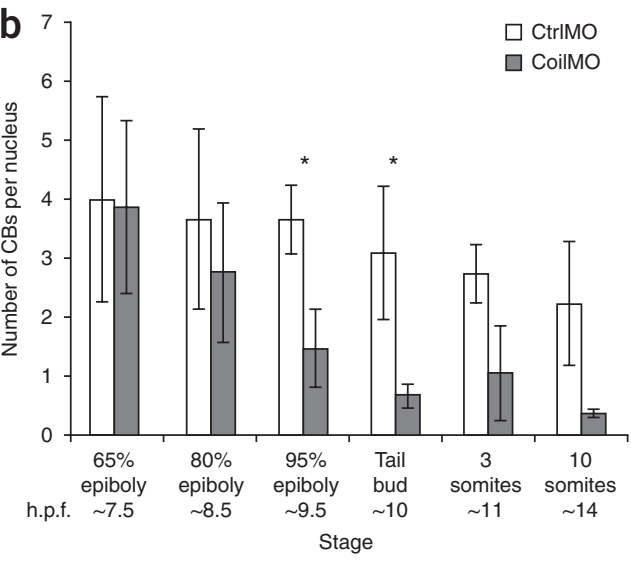

The intensity of coilin staining decreased upon CoilMO injection, beginning at $\sim 6$ h.p.f., and became nearly undetectable by $\sim 11$ h.p.f.; western blotting revealed a marked loss of coilin protein in CoilMO-injected embryos at 14 h.p.f. (Supplementary Fig. 3). As in other organisms, spliceosomal snRNPs are concentrated in zebrafish CBs marked by coilin ${ }^{30}$. In CoilMO-injected embryos (morphants), snRNPs were dispersed throughout the nucleoplasm (Fig. 2a and Supplementary Fig. 4a). The number of snRNPcontaining foci per nucleus in the coilin morphants was significantly decreased by a factor of 5 on average (Fig. 2b), consistent with a requirement for coilin in $\mathrm{CB}$ integrity ${ }^{23,24}$. Notably, embryos rescued by co-injection with YFP-coilin showed CBs defined by YFP-coilin protein and endogenous snRNPs (Fig. $2 \mathbf{b}$ ). The number of CBs in coilin morphants at 10 h.p.f. was completely restored to CtrlMO levels by injection of YFP-coilin mRNA (see Fig. 2b legend). This indicates that YFP-coilin expression rescued $\mathrm{CB}$ morphology in coilin morphants. Therefore, the presence of $\mathrm{CBs}$ containing coilin and the splicing machinery correlates with embryonic viability.

To determine whether CB dispersal paralleled the loss of coilin and to relate $\mathrm{CB}$ number to other observed phenotypes in CoilMOinjected embryos, we quantified CB numbers in three dimensions

Figure 4 Coilin morphants show defects in snRNP assembly and expression of spliced mRNAs. (a) Metabolic labeling of snRNAs in embryos co-injected with [ $\alpha-32$ P]UTP and CoilMO (MO) or CtrIMO (C) grown at $28^{\circ} \mathrm{C}$ for $\sim 14 \mathrm{~h}$. A representative gel shows the positions of $\mathrm{U} 1$, U2, U4 and U5 snRNAs immunoprecipitated with anti-Sm antibody (Y12) via assembled Sm cores. Expression of tRNA and U1 and U2 snRNA was not affected by coilin depletion, as indicated in the input lanes. Right, CoilMO-induced decreases in assembled snRNPs are revealed through quantification of each snRNA recovered in the Y12 immunoprecipitate. CoilMO relative to CtrIMO is shown in the histogram (mean \pm s.e.m., $n=5$ experiments). U6 snRNA was not detectable by metabolic labeling, perhaps reflecting abundant maternal stores of U6 snRNP as observed in Xenopus laevis ${ }^{46}$. (b) Abundance of spliced mRNA by quantitative RT-PCR detected by primers spanning the indicated splice junctions. Total RNA was isolated from 5- to 6-somite-stage embryos injected with 1 pmol CoilMO with and without human snRNPs or else injected with 1 pmol CtrlMO. The levels are given relative to control (CtrIMO-injected embryos) and normalized to 5S RNA for $n=3$ experiments. Error bars, s.e.m.
Figure 3 U4 snRNA localization in living embryos reveals timing of CB dispersal upon coilin depletion. (a) Embryos co-injected with Alexa488-labeled U4 snRNA ( $3 \mathrm{fmol}$ ) and CoilMO ( 1 pmol) and imaged in vivo at tailbud stage ( 10 h.p.f.) show CB dispersal. Nuclei are outlined in single confocal sections for clarity. Arrowheads indicate U4-positive CBs. Cytoplasmic signal may correspond to U bodies, where excess injected snRNA may accumulate ${ }^{32,45}$. Note that the gain in the CoilMO image is set higher than in the CtrIMO panel, to capture the dispersed nucleoplasmic signal. (b) Quantification of CB number in vivo with Alexa-488-U4 snRNA identifies the time of CB dispersal in coilin morphants. Asterisks indicate statistical significance (two-sample $t$-test, $P=0.01$ for $95 \%$ epiboly; $P=0.02$ for tailbud, mean \pm s.d., $n=3$ independent experiments).

in living embryos to yield an absolute number of CBs per nucleus. To accomplish this, we injected fluorescently labeled U4 snRNA, which assembles into snRNPs and concentrates rapidly in CBs in vivo ${ }^{30,32}$, for use as a marker of CBs (Fig. 3). We used threedimensional rendering followed by automatic detection and counting of U4 snRNA-positive nuclear foci to determine the number of CBs per nucleus in embryos injected with CtrlMO or CoilMO. The analysis revealed a progressive loss of CBs in CoilMO-injected embryos beginning at $\sim 9$ h.p.f. (Fig. 3b), 5 h before we detected cell-proliferation defects (Supplementary Fig. 2). Reduction in CB number paralleled the loss of coilin protein, which was nearly undetectable by $\sim 14$ h.p.f. (Supplementary Fig. 3). Consistent with previous reports from tissue-culture systems ${ }^{20,23}$, U85 scaRNA and cyan fluorescent protein (CFP)-SMN continued to localize in residual bodies in coilin morphants at the same stage (Supplementary Figs. 4 b and 5). Thus, coilin depletion leads to dispersal of CBs before cell-proliferation defects and developmental arrest.

\section{Defects in snRNP biogenesis and splicing in the coilin morphant} The disruption of spliceosomal snRNP localization due to coilin depletion prompted us to ask whether splicing functions were compromised. To determine whether coilin is required for snRNP biogenesis, we carried out metabolic labeling of zygotically transcribed snRNAs at 0-14 h.p.f. in control and coilin morphants, followed by immunoprecipitation with anti-Sm and gel electrophoresis. We detected a $\sim 50 \%$ reduction in de novo-assembled snRNPs in coilin morphants (Fig. 4a). Note that expression of tRNA, snRNAs and the Sm proteins was unaffected by CoilMO injection (Fig. 4a and Supplementary Fig. 6). The observed decrease in snRNP production upon coilin depletion might be expected to compromise levels of spliced mRNAs in embryos. We did not expect a complete block of gene expression, because direct inhibition of splicing leads to developmental arrest at 4.5 h.p.f., shortly after $\mathrm{ZGA}^{33}$; coilin morphants
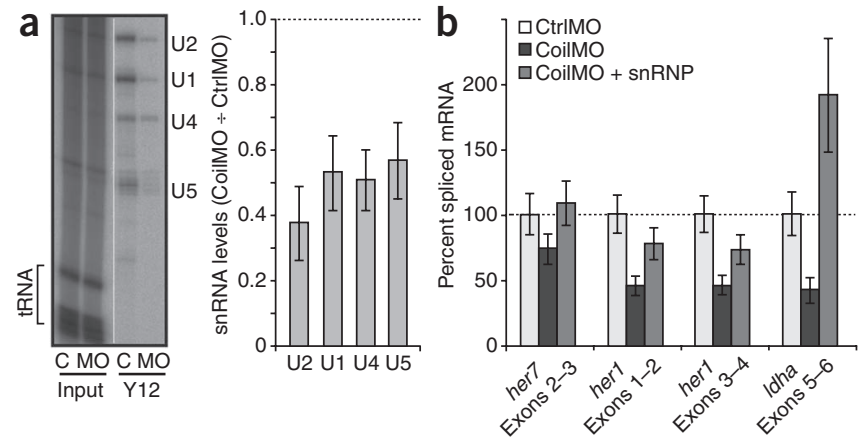
Figure 5 Timeline of morphological, cellular and molecular defects in embryos depleted of coilin. Reduction of coilin protein upon knockdown is depicted according to qualitative impressions from immunostaining (see Supplementary Fig. 3) and the decrease in $C B$ number reflects quantification shown in Figure 3. Time points at which defects in mRNA expression (Fig. 4), cell proliferation and apoptosis (Supplementary Fig. 2) were detected are shown.

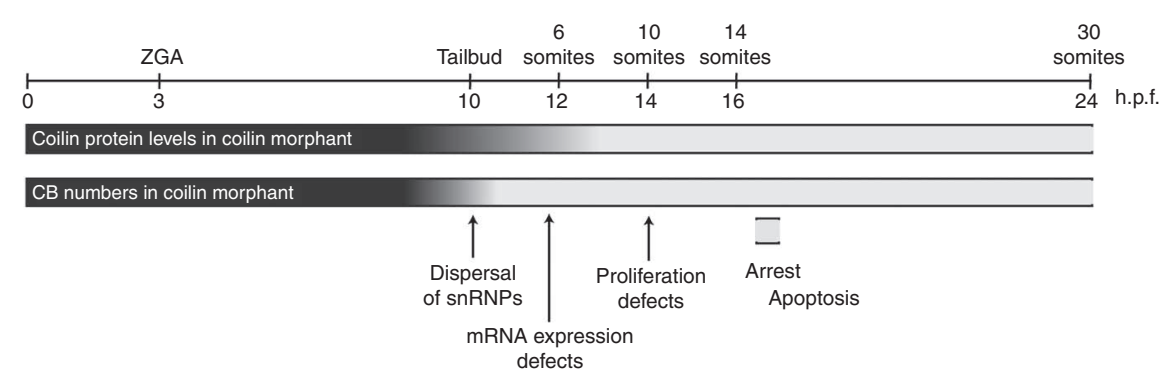

live for $\sim 18 \mathrm{~h}$, and their snRNP biogenesis is reduced, not abolished. RT-PCR analysis, using primers spanning a variety of splice junctions in representative transcripts, detected quantitative losses (25-50\%) of spliced mRNAs in coilin morphant embryos at $\sim 12$ h.p.f. (Fig. 4b). Thus, we observed mRNA losses after the observed dispersal of CBs at the morphological level and before developmental arrest. These molecular phenotypes are summarized in the context of the other changes due to coilin depletion in a developmental timeline in Figure 5. The observed defects in snRNP biogenesis and spliced mRNA levels suggest that deficits in splicing are linked to subsequent failure in cell proliferation and survival of the embryo.

Mature human spliceosomal snRNPs rescue the coilin morphant To determine whether the observed mRNA losses were due to reduced levels of assembled snRNPs in coilin morphant embryos, we sought to complement this molecular phenotype by injecting splicing-competent snRNPs purified by TMG chromatography from human cells. Note that human coilin was not detectable in these preparations (Supplementary Fig. 7 and ref. 4). Co-injection of snRNPs led to a complete rescue of spliced mRNA levels (Fig. 4b). Although intron-retained transcripts are known to be unstable, we detected increased levels of unspliced transcripts in

a

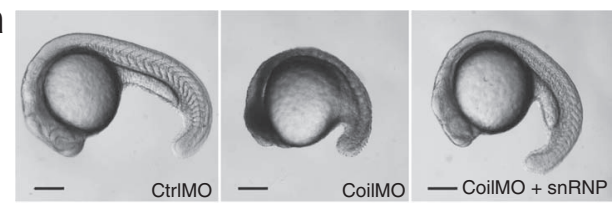

b

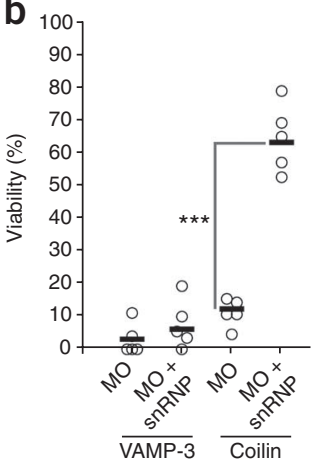

d CtrIMO

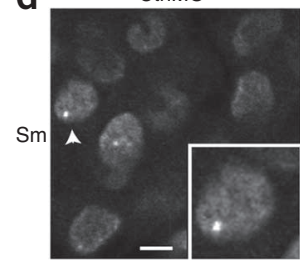

C 100

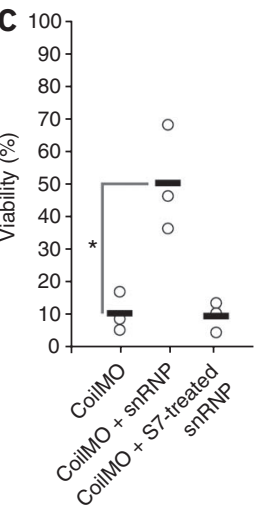

CoilMO

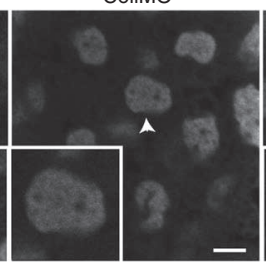

CoilMO + snRNP

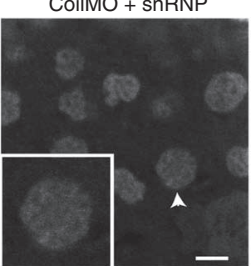

CoilMO-injected embryos, and this effect was also counteracted by injection of mature human snRNPs (Supplementary Fig. 8). These data indicate that purified human snRNPs are catalytically active in zebrafish embryos, consistent with a prior study ${ }^{34}$. Moreover, rescue of mRNA levels by human snRNPs confirms that the mRNA-loss in coilin morphants was caused specifically by the snRNP deficit. We conclude that coilin is required to generate sufficient levels of snRNPs for zygotic mRNA expression.

Reduced expression of the zygotic genome provides a logical explanation for the failure of coilin morphants to proliferate and develop (Fig. 5). Is the deficit in functional snRNPs the cause of embryonic lethality? Notably, co-injection of purified human snRNPs rescued the lethality caused by CoilMO (Fig. 6a). The viability of embryos injected with both CoilMO and snRNPs was significantly increased, from $\sim 10 \%$ (CoilMO alone) to $\sim 65 \%$ on average (Fig. 6b). Note that viability in a number of the snRNP-injected groups reached $100 \%$ or close to it (see below); many of the rescued embryos survived for 1 week (at which point we terminated the experiment), indicating that complementation by snRNPs allowed the embryos to properly complete development. We expected lower levels of rescue in some experimental groups due to the mosaic distribution of injected materials among cells of the embryo ${ }^{35}$. Rescue was specific to coilin morphants, because snRNP injection did not rescue early embryonic lethality caused by a distinct morpholino targeting an endocytic pathway-related molecule, VAMP3 (ref. 36) (Fig. 6b). Moreover, intact snRNAs were required, because prior S7 nuclease digestion of the snRNP preparation completely blocked rescue (Fig. 6c). Thus, elevating the levels of snRNP proteins alone is insufficient to complement the CoilMO phenotype. Finally, and in contrast to the rescue attained by YFP-coilin (Fig. 2b and Supplementary Fig. 9), snRNP injection did not lead to reconstitution of nuclear foci in the morphant (Fig. 6d and Supplementary Fig. 9), consistent with a requirement for coilin

Figure 6 Purified human snRNPs rescue the viability of coilin morphants. (a) Zebrafish embryos injected with purified human snRNPs or BSA, followed by 1 pmol of either CtrlMO or CoilMO at 24 h.p.f. Scale bar, $250 \mu \mathrm{m}$. (b) Groups of 20-40 embryos were injected with 300 pg BSA (MO) or purified human snRNPs (MO + snRNP) and then with 1 pmol CoilMO or VAMP3-MO. After $24 \mathrm{~h}$ at $28^{\circ} \mathrm{C}$, independent groups of embryos were scored for viability (open circles). Asterisks, significance at $P<0.001$ (two-sample $t$-test, unequal variance, \pm s.e.m., $n=5$ ). (c) Groups of 20-40 embryos were injected with 300 pg BSA (CoilMO), purified human snRNPs (CoilMO + snRNP) or S7 nuclease-treated snRNPs (CoilMO + S7-treated snRNP), followed by 1 pmol CoilMO. Asterisks, significance at $P=0.03$ (two-sample $t$-test, unequal variance, \pm s.d., $n=3$ ). In $\mathbf{b}$ and $\mathbf{c}$, average viability in the experimental groups is plotted as a horizontal black bar. (d) Injection of purified human snRNPs does not cause nuclear body formation. Coilin morphant embryos at $\sim 10$ h.p.f., with or without human snRNP injection stained with anti-Sm (Y12) antibody. Nuclear bodies were detected only in control embryos. Single confocal sections are shown. Arrowheads indicate nuclei magnified in the insets $(2 \times)$. Scale bars, $10 \mu \mathrm{m}$. 
Figure 7 Individual mature human snRNPs bypass the need for coilin, and snRNAs are not sufficient. Groups of 20-40 fertilized 1-cell embryos were injected with CoilMO (1 pmol) with or without different preparations of snRNAs, spliceosomal snRNPs or CtrIMO. After $24 \mathrm{~h}$ of development at $28^{\circ} \mathrm{C}$, independent groups of embryos were scored for percent viability (open circles). The preparations include total snRNPs (orange panel), U1 (green panel), U2 12S and U2 17S (blue panel) and U4/U5•U6 tri-snRNPs (yellow panel). Total human spliceosomal snRNPs were purified from HeLa cells by chromatography on anti-TMG columns, and individual snRNPs were each further purified by glycerol-gradient centrifugation and ion-exchange chromatography. Native, endogenously modified U1 and U2 snRNAs were extracted from these preparations and purified; unmodified, in vitro-transcribed U1 and U2 snRNAs were injected for comparison (green and blue panels, as indicated). The snRNA and

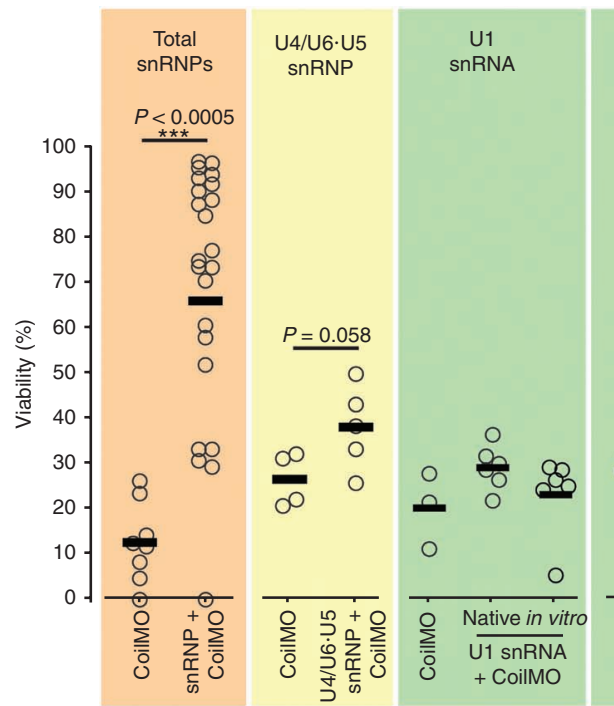

snRNP preparations were calibrated for injection of the same amount of the respective snRNA. Average values are represented by horizontal black bars; individual data points are shown as open circles. Where differences were statistically significant, $P$ values are shown on the graph (two-sample $t$-test, unequal variance, \pm s.d., $n \geq 3$ experiments as indicated on graph).

in $\mathrm{CB}$ assembly ${ }^{23,24}$. The fact that injection of purified human snRNPs bypasses the embryonic requirement for coilin indicates that a deficit in snRNP assembly is the primarily lesion caused by coilin depletion.

To further dissect the embryonic requirement for assembled snRNPs in zygotic gene expression, we tested individual snRNPs and snRNP intermediates for their ability to rescue coilin morphants. We further purified U1, U2 and U4/U6•U5 snRNPs from the total snRNP preparation by glycerol-gradient centrifugation ${ }^{37}$ and ion-exchange chromatography. Supplementary Figure 7 shows the purity of the fractions by RNA and protein gel electrophoresis. U1 snRNP injection did not rescue, suggesting that U1 snRNP levels in coilin morphants are sufficient for splicing (Fig. 7). In contrast, the viability of coilin morphants and the extent of survival among arrested embryos were significantly increased upon coinjection of purified U2 snRNP or U4/U6 65 snRNP alone (Fig. 7 and Supplementary Fig. 10). Notably, U2 snRNPs were nearly as potent as total snRNPs. Therefore, we presume that snRNP levels in coilin morphants are reduced below a threshold concentration, which can be at least partially overcome by increasing the concentration of just one snRNP.

The robust activity of purified human U2 snRNP in the coilin morphant rescue assay allowed us to test whether U2 snRNA is sufficient. All snRNAs are capped at their $5^{\prime}$ ends by TMG, and internal bases are modified by scaRNAs present in the $\mathrm{CB}^{7}$. Internally modified nucleotides are required for U2 snRNP function, whereas the TMG cap is not ${ }^{38}$. Therefore, we tested modified U1 and U2 snRNAs purified from mature human snRNPs in our assay (Supplementary Fig. 7). Note that modified and unmodified U2 snRNAs assemble equally well into U2 snRNPs in vitro ${ }^{39}$ and that human and zebrafish U1 and U2 snRNAs are $92 \%$ and 91\% identical, respectively. Neither in vitro-transcribed nor native U1 or U2 snRNAs was sufficient to rescue viability or partial development (Fig. 7 and Supplementary Fig. 10). Thus, the coilin morphant phenotype is not due to a failure to transcribe or post-transcriptionally modify U2 snRNA. Instead, later steps in snRNP assembly, involving the assembly of proteins onto snRNAs, are implicated.

\section{DISCUSSION}

In this study, we have taken advantage of zebrafish embryology and imaging to functionally test the contribution of CBs to gene expression. We disrupted morphologically defined CBs in embryos by depleting coilin, a protein that is absolutely required for the assembly and maintenance of the $\mathrm{CB}^{23,24}$. Coilin depletion led to reduced cell proliferation, developmental arrest and cell death. Before we detected these defects, we detected CB disruption and the dispersal of spliceosomal snRNPs, leading us to hypothesize that lethality may be due to defective splicing levels in embryos depleted of coilin. Indeed, we observed increased intron retention and reduced mRNA levels for several transcripts tested in the morphants. This is consistent with a recent report of splicing defects upon coilin knockdown in mammalian tissue-culture cells ${ }^{40}$. Furthermore, both splicing and embryonic lethality were fully rescued by injecting purified mature human snRNPs. These observations indicate that coilin-depleted embryos specifically arrest due to reduced snRNP function. Notably, injection of mature human snRNPs did not reconstitute snRNP-enriched nuclear bodies, showing that rescue was not due to the recovery of some other potential function of CBs. Taken together, these data show that coilin is required for $\mathrm{CB}$ integrity and splicing in zebrafish embryos, supporting embryonic gene expression and growth beyond the segmentation stage.

The following observations show that a failure in snRNP assembly upon coilin depletion is the primary cause of splicing deficits and loss of viability. First, we observed by metabolic labeling a $\sim 50 \%$ reduction in the levels of snRNPs produced de novo after snRNP dispersal by coilin depletion. Notably, overall levels of snRNAs and Sm proteins were unaffected. Second, rescue of splicing and viability by injection of human snRNPs was abolished when snRNAs had been previously digested by S7 nuclease. This shows that elevated levels of injected snRNP proteins were unable to compensate for the loss of coilin. Third, injection of in vitro-transcribed or native, modified U1 or U2 snRNAs were unable to rescue, indicating that, although snRNAs are required, they are not sufficient to rescue the splicing deficit induced by coilin depletion. This indicates that $\mathrm{CB}$ integrity is not required for snRNA modification, consistent with the 
recent finding in flies that, although coilin is required for scaRNA concentration in nuclear bodies, snRNA modification appears to be normal in the absence of coilin ${ }^{41}$. Fourth, highly purified, fully assembled $\mathrm{U} 2$ or $\mathrm{U} 4 / \mathrm{U} 6 \bullet \mathrm{U} 5$ tri-snRNP was capable of significant rescue of the CoilMO phenotype, whereas U1 snRNP was not. This suggests that stable U2 snRNP interactions with pre-mRNA, which trigger spliceosome assembly ${ }^{6}$, represent a rate-limiting step in embryonic gene expression. Because neither the snRNP proteins nor the snRNAs alone had rescuing ability and only mature assembled snRNPs were active, we conclude that macromolecular assembly of snRNPs is the essential function of coilin in zebrafish embryos.

We attribute the lethality of coilin depletion to defects in late stages of snRNP biogenesis. After core snRNPs are reimported to the nucleus and snRNAs are modified, final steps of maturation must occur before snRNPs are competent for splicing; these include RNA structural rearrangements and recruitment of additional snRNPspecific proteins ${ }^{5}$. For example, the U4/U6•U5 tri-snRNP is formed from three individual snRNPs (U4, U5 and U6) that are inactive for splicing before assembly. A transient intermediate, the U4/U6 di-snRNP, is highly concentrated in CBs and accumulates there when $\mathrm{U} 4 / \mathrm{U} 6 \bullet \mathrm{U} 5$ tri-snRNP formation is blocked ${ }^{14-16}$. Partial rescue of the coilin morphant by the U4•U5/U6 snRNP implicates coilin in these late stages of assembly. Notably, U1 snRNP did not rescue the coilin morphant phenotype. U1 is the least well-concentrated snRNA in CBs, and mature U1 snRNP does not target to CBs in nuclear transport reactions, whereas U2 snRNP does ${ }^{12,42}$. Similarly to the tri-snRNP, intermediates in mature U2 snRNP formation can be trapped in CBs upon knockdown of appropriate assembly factors ${ }^{17}$. $\mathrm{U} 2$ snRNP is present in cells in $12 \mathrm{~S}$ and $17 \mathrm{~S}$ forms, reflecting the association of the core U2 snRNP containing the Sm ring U2A and U2B proteins (12S) with the SF3 complex (17S $)^{5,6}$. Notably, both the $12 \mathrm{~S}$ and $17 \mathrm{~S}$ forms of the $\mathrm{U} 2 \mathrm{snRNP}$ rescued coilin morphants equally, indicating that formation of the 12 S U2 snRNP is compromised upon coilin depletion. The present findings provide an organismal context for prior observations showing the involvement of CBs in snRNP assembly. Moreover, they show that coilin is required for sufficient levels of snRNP biogenesis in the embryo.

How does coilin promote snRNP assembly? Spliceosomal snRNPs explore the nuclear volume by Brownian diffusion ${ }^{3,15,43}$. Given this, the rate at which immature snRNPs encounter partner proteins or other snRNPs will determine how quickly their assembly occurs. One possibility is that targeting of immature snRNPs to CBs promotes interactions among potential binding partners by creating a high local concentration of each partner. The snRNP concentration in CBs is $\sim 20$ times the nucleoplasmic levels in HeLa cells, establishing a dramatic concentration difference that increases the likelihood of diffusive interactions ${ }^{32}$. When snRNP assembly is modeled according to random walk-and-capture behavior, simulations predict that CBs enhance the rate of snRNP assembly by a factor of $\sim 10$ in HeLa cells ${ }^{32}$. If this is true, dispersal of CBs should lead to a reduced rate of snRNP assembly. Consistent with this, we observed a $\sim 50 \%$ reduction in the levels of newly assembled snRNPs upon coilin depletion in embryos, despite the continued expression of snRNP proteins and snRNAs. Note that the continued presence of maternally provided CBs during ZGA ( $\sim 3$ h.p.f.) likely contributed to snRNP assembly dynamics before CB dispersal by CoilMO treatment. Therefore, the true effect of CBs on the rate of snRNP assembly is probably higher than a factor of 2. Related to this, rescue of the morphant by injection of YFPcoilin was accompanied by re-formation of CBs, whereas injection of human snRNPs was not (Figs. 2 b and 6d and Supplementary Fig 9). This underscores the notion that the embryonic requirement is for
snRNPs and not CBs per se. Thus, the simplest explanation for the embryonic requirement for coilin in vertebrates is that the failure to concentrate assembly intermediates leads to a decrease in the rate of protein-protein and RNA-protein interactions required for efficient snRNP assembly.

In addition to spliceosomal snRNPs, four other distinct sets of RNA processing machineries - those supporting histone mRNA $3^{\prime}$-end processing, telomere maintenance, rRNA and miRNA processing-are highly enriched in CBs in some cells and organisms ${ }^{1,7}$. However, the histone mRNA $3^{\prime}$-processing machinery is concentrated in a separate, coilin-deficient nuclear body in zebrafish embryos, suggesting that defects in histone expression are unlikely to contribute to the coilin morphant phenotype ${ }^{30}$. Because purified spliceosomal snRNPs were able to restore viability of coilin morphants, it appears that other RNA-processing machineries are not critically compromised by coilin depletion in zebrafish embryos. Although involvement of CBs in these processes may be required in other contexts, we propose that the rapid deployment of zygotic gene expression programs in the early zebrafish sensitizes the embryo to a reduction in the rate of snRNP biogenesis. We would expect a similar sensitivity to loss of coilin in other rapidly developing systems with a reliance on splicing. Although coilin mutants in flies and plants are viable ${ }^{19,21}$, coilin gene disruption in the mouse is embryonic semilethal ${ }^{29}$. Our present findings predict that homozygous coilin mutant mouse embryos may be less fit due to a failure to splice.

This work reveals a basic principle in cellular logistics, in which coilin facilitates macromolecular assembly by concentrating interacting components in subcellular compartments without the diffusional barrier of membranes. Recently, P granules in Caenorhabditis elegans - known determinants of germ cell fate-were shown to possess physicochemical properties of liquid droplets, suggesting that compartments lacking membranes reflect an intrinsic tendency to self-assemble through networks of low-affinity intermolecular interactions ${ }^{44}$. Coilin self-associates to produce a multivalent scaffold, and it binds snRNPs and other CB components with low affinity ${ }^{5,26,27}$. As a consequence, $\mathrm{CB}$ components exchange rapidly with nucleoplasm, where mature snRNPs are active ${ }^{3}$. Based on our findings in zebrafish embryos, we propose that coilin's role as a primary node in the $\mathrm{CB}$ interaction network is essential for efficient macromolecular assembly of spliceosomal snRNPs.

\section{METHODS}

Methods and any associated references are available in the online version of the paper at http://www.nature.com/nsmb/.

Note: Supplementary information is available on the Nature Structural \& Molecular Biology website.

\section{ACKNOWLEDGMENTS}

The authors thank D. Stanek, J. Geiger and C.-P. Heisenberg for their early involvement in this project and comments on the manuscript, K. Simons and M. Zerial for helpful discussions, G. Morris (Keele Univ.) for the gift of $1 F 1$ antibody, I. Öchsner for her help with snRNA preparations, and the Max Planck Institute of Molecular Cell Biology fish facility. This work was supported by the Max Planck Society (K.M.N., A.C.O. and R.L.) and grants from the German Research Foundation (NE909/2-1 to K.M.N.) and the European Commission (EURASNET-518238 to K.M.N. and R.L.).

\section{AUTHOR CONTRIBUTIONS}

M.S., R.L., A.C.O. and K.M.N. designed the experiments; S.T. and G.W. purified snRNPs and snRNAs; M.S. and K.M.N. performed the experiments; M.S. analyzed the data; M.S., A.C.O. and K.M.N. wrote the paper.

\section{COMPETING FINANCIAL INTERESTS}

The authors declare no competing financial interests. 
Published online at http://www.nature.com/nsmb/.

Reprints and permissions information is available online at http://npg.nature.com/ reprintsandpermissions/.

1. Matera, A.G., Izaguire-Sierra, M., Praveen, K. \& Rajendra, T.K. Nuclear bodies: random aggregates of sticky proteins or crucibles of macromolecular assembly? Dev. Cell 17, 639-647 (2009).

2. Gall, J.G. Cajal bodies: the first 100 years. Annu. Rev. Cell Dev. Biol. 16, 273-300 (2000).

3. Dundr, M. et al. In vivo kinetics of Cajal body components. J. Cell Biol. 164, 831-842 (2004)

4. Makarov, E.M. et al. Small nuclear ribonucleoprotein remodeling during catalytic activation of the spliceosome. Science 298, 2205-2208 (2002).

5. Stanek, D. \& Neugebauer, K.M. The Cajal body: a meeting place for spliceosomal snRNPs in the nuclear maze. Chromosoma 115, 343-354 (2006).

6. Wahl, M.C., Will, C.L. \& Lührmann, R. The spliceosome: design principles of a dynamic RNP machine. Cell 136, 701-718 (2009).

7. Kiss, T. Biogenesis of small nuclear RNPs. J. Cell Sci. 117, 5949-5951 (2004).

8. Meister, G., Buhler, D., Pillai, R., Lottspeich, F. \& Fischer, U. A multiprotein complex mediates the ATP-dependent assembly of spliceosomal U snRNPs. Nat. Cell Biol. 3, 945-949 (2001).

9. Narayanan, U., Achsel, T., Luhrmann, R. \& Matera, A.G. Coupled in vitro import of U snRNPs and SMN, the spinal muscular atrophy protein. Mol. Cell 16, 223-234 (2004).

10. Fischer, U., Sumpter, V., Sekine, M., Satoh, T. \& Luhrmann, R. Nucleo-cytoplasmic transport of $U$ snRNPs: definition of a nuclear location signal in the Sm core domain that binds a transport receptor independently of the m3G cap. EMBO J. 12, 573-583 (1993).

11. Sleeman, J.E. \& Lamond, A.I. Newly assembled snRNPs associate with coiled bodies before speckles, suggesting a nuclear snRNP maturation pathway. Curr. Biol. 9, 1065-1074 (1999).

12. Ospina, J.K. et al. Cross-talk between snurportin1 subdomains. Mol. Biol. Cell 16, 4660-4671 (2005).

13. Jady, B.E. et al. Modification of Sm small nuclear RNAs occurs in the nucleoplasmic Cajal body following import from the cytoplasm. EMBO J. 22, 1878-1888 (2003).

14. Stanek, D. \& Neugebauer, K.M. Detection of snRNP assembly intermediates in Cajal bodies by fluorescence resonance energy transfer. J. Cell Biol. 166, 1015-1025 (2004).

15. Stanek, D. et al. Spliceosomal small nuclear ribonucleoprotein particles repeatedly cycle through Cajal bodies. Mol. Biol. Cell 19, 2534-2543 (2008).

16. Schaffert, N., Hossbach, M., Heintzmann, R., Achsel, T. \& Luhrmann, R. RNAi knockdown of hPrp31 leads to an accumulation of U4/U6 di-snRNPs in Cajal bodies. EMBO J. 23, 3000-3009 (2004).

17. Nesic, D., Tanackovic, G. \& Kramer, A. A role for Cajal bodies in the final steps of U2 snRNP biogenesis. J. Cell Sci. 117, 4423-4433 (2004).

18. Handwerger, K.E., Murphy, C. \& Gall, J.G. Steady-state dynamics of Cajal body components in the Xenopus germinal vesicle. J. Cell Biol. 160, 495-504 (2003).

19. Collier, S. et al. A distant coilin homologue is required for the formation of Cajal bodies in Arabidopsis. Mol. Biol. Cell 17, 2942-2951 (2006).

20. Lemm, I. et al. Ongoing $U$ snRNP biogenesis is required for the integrity of Cajal bodies. Mol. Biol. Cell 17, 3221-3231 (2006).

21. Liu, J.L. et al. Coilin is essential for Cajal body organization in Drosophila melanogaster. Mol. Biol. Cell 20, 1661-1670 (2009).

22. Stanek, D., Rader, S.D., Klingauf, M. \& Neugebauer, K.M. Targeting of U4/U6 small nuclear RNP assembly factor SART3/p110 to Cajal bodies. J. Cell Biol. 160, 505-516 (2003)
23. Tucker, K.E. et al. Residual Cajal bodies in coilin knockout mice fail to recruit Sm snRNPs and SMN, the spinal muscular atrophy gene product. J. Cell Biol. 154, 293-307 (2001).

24. Kaiser, T.E., Intine, R.V. \& Dundr, M. De novo formation of a subnuclear body. Science 322, 1713-1717 (2008)

25. Tucker, K.E. et al. Structure and characterization of the murine $\mathrm{p} 80$ coilin gene Coil. J. Struct. Biol. 129, 269-277 (2000)

26. Hebert, M.D. \& Matera, A.G. Self-association of coilin reveals a common theme in nuclear body localization. Mol. Biol. Cell 11, 4159-4171 (2000).

27. $\mathrm{Xu}, \mathrm{H}$. et al. The $\mathrm{C}$-terminal domain of coilin interacts with $\mathrm{Sm}$ proteins and U snRNPs. Chromosoma 114, 155-166 (2005).

28. Hebert, M.D. \& Matera, A.G. Self-association of coilin reveals a common theme in nuclear body localization. Mol. Biol. Cell 11, 4159-4171 (2000).

29. Walker, M.P., Tian, L. \& Matera, A.G. Reduced viability, fertility and fecundity in mice lacking the cajal body marker protein, coilin. PLoS One 4, e6171 (2009).

30. Strzelecka, M., Oates, A.C. \& Neugebauer, K.M. Dynamic control of Cajal body number during zebrafish embryogenesis. Nucleus 1, 96-108 (2010).

31. Thisse, B. \& Thisse, C. Fast release clones: a high throughput expression analysis. ZFIN Direct Data Submission (ZFIN, 2004).

32. Klingauf, M., Stanek, D. \& Neugebauer, K.M. Enhancement of U4/U6 small nuclea ribonucleoprotein particle association in Cajal bodies predicted by mathematical modeling. Mol. Biol. Cell 17, 4972-4981 (2006)

33. Konig, H., Matter, N., Bader, R., Thiele, W. \& Muller, F. Splicing segregation: the minor spliceosome acts outside the nucleus and controls cell proliferation. Cell 131, 718-729 (2007)

34. Winkler, C. et al. Reduced U snRNP assembly causes motor axon degeneration in an animal model for spinal muscular atrophy. Genes Dev. 19, 2320-2330 (2005).

35. McClintock, J.M., Kheirbek, M.A. \& Prince, V.E. Knockdown of duplicated zebrafish hoxb1 genes reveals distinct roles in hindbrain patterning and a novel mechanism of duplicate gene retention. Development 129, 2339-2354 (2002).

36. McMahon, H.T. et al. Cellubrevin is a ubiquitous tetanus-toxin substrate homologous to a putative synaptic vesicle fusion protein. Nature 364, 346-349 (1993).

37. Bach, M., Bringmann, P. \& Luhrmann, R. Purification of small nuclear ribonucleoprotein particles with antibodies against modified nucleosides of small nuclear RNAs. Methods Enzymol. 181, 232-257 (1990).

38. Dönmez, G., Hartmuth, K. \& Lührmann, R. Modified nucleotides at the $5^{\prime}$ end of human U2 snRNA are required for spliceosomal E-complex formation. RNA 10, 1925-1933 (2004).

39. Segault, V., Will, C.L., Sproat, B.S. \& Luhrmann, R. In vitro reconstitution of mammalian U2 and U5 snRNPs active in splicing: Sm proteins are functionally interchangeable and are essential for the formation of functional U2 and U5 snRNPs. EMBO J. 14, 4010-4021 (1995)

40. Whittom, A.A., Xu, H. \& Hebert, M.D. Coilin levels and modifications influence artificial reporter splicing. Cell. Mol. Life Sci. 65, 1256-1271 (2008).

41. Deryusheva, S. \& Gall, J.G. Small Cajal body-specific RNAs (scaRNAs) of Drosophila function in the absence of Cajal bodies. Mol. Biol. Cell 20, 5250-5259 (2009).

42. Matera, A.G. \& Ward, D.C. Nucleoplasmic organization of small nuclear ribonucleoproteins in cultured human cells. J. Cell Biol. 121, 715-727 (1993).

43. Rino, J. et al. A stochastic view of spliceosome assembly and recycling in the nucleus. PLoS Comput. Biol. 3, 2019-2031 (2007).

44. Brangwynne, C.P. et al. Germline P granules are liquid droplets that localize by controlled dissolution/condensation. Science 324, 1729-1732 (2009).

45. Liu, J.L. \& Gall, J.G. U bodies are cytoplasmic structures that contain uridine-rich small nuclear ribonucleoproteins and associate with $\mathrm{P}$ bodies. Proc. Natl. Acad. Sci. USA 104, 11655-11659 (2007).

46. Hamm, J. An abundant U6 snRNP found in germ cells and embryos of Xenopus laevis embryos. EMBO J. 8, 4179-4187 (1989). 


\section{ONLINE METHODS}

Fish maintenance. We maintained and harvested zebrafish $\mathrm{AB}$ strain adults and embryos as previously described ${ }^{47}$.

Vectors. We cloned constructs for in vitro transcription of mRNAs encoding YFP-coilin and SMN-CFP by standard methods. Zebrafish U4 snRNA in TOPO vector was a gift from A. Bindereif (Justus-Liebig-Univ., Giessen, Germany). U4 snRNA in pSP65 was described previously ${ }^{48}$. Human U85 scaRNA plasmid was a gift of A.M. Kiss.

In vitro transcription. We used mMESSAGE mMACHINE SP6 Kit (Ambion) for mRNA synthesis and MEGAshortscript T7 Kit for small-RNA synthesis (Ambion). We carried out the synthesis of fluorescently labeled human U4 snRNA as previously described ${ }^{32}$.

Microinjections. We injected embryos into the yolk or the 1-cell blastoderm with $\sim 1$ nl of material following standard methods ${ }^{49}$. Morpholinos (Gene Tools LLC) had the following sequences $\left(5^{\prime} \rightarrow 3^{\prime}\right)$ : CoilMO, GCCATGCCTGAACACTACACT ACAG; CoilMO-splice, GACATTCATCTCTCACCTGTAAAAT; standard control (CtrlMO), CCTCTTACCTCAGTTACAATTTATA.

We validated the coilin mRNA 5'-UTR target sequence retrieved from GenBank by cloning and sequencing the corresponding genomic fragment. We dissolved morpholinos in Danieu's buffer. Before each injection, we incubated morpholino stock solution at $65^{\circ} \mathrm{C}$ for $5 \mathrm{~min}$, snap-cooled it on ice and adjusted its concentration to $1 \mathrm{mM}$. We injected in vitro-transcribed mRNAs and fluorescently labeled RNAs in $100 \mathrm{mM} \mathrm{KCl,} 5$ mM Tris- $\mathrm{HCl}, \mathrm{pH}$ 7.4, 0.25 mM EDTA ${ }^{49}$.

Human snRNP and snRNA preparations. We tested 12 batches of human spliceosomal snRNP preparations. We isolated three independent preparations from HeLa cell nuclear extract by anti- $\mathrm{m}_{3}{ }^{2,2,7} \mathrm{G}$ immunoaffinity chromatography ${ }^{37}$ and eluted them in buffer containing $250 \mathrm{mM}$ salt. We also eluted another three preparations in a buffer with $420 \mathrm{mM}$ salt. For each set, we exchanged buffer to PBS by dialysis or on a buffer exchange column. We further fractionated anti- $\mathrm{m}_{3} \mathrm{G}$ immunoaffinity chromatography eluates into U1, U2 and U4/U6•U5 components by glycerol-gradient centrifugation and ion-exchange chromatography. We reconstituted 17S U2 snRNP from purified 12S U2 snRNP and high-salt immunoaffinity-purified SF3a and SF3 $b^{50,51}$. We buffer-exchanged U4/U6•U5 tri-snRNP to high-salt PBS ( $1 \mathrm{M} \mathrm{NaCl})$. We exchanged all other snRNP fractions to PBS by buffer-exchange columns. We concentrated tri-snRNP and 12S U2 snRNP fractions by ultrafiltration (Vivaspin concentrators, Sartorius). We extracted native snRNAs from spliceosomal snRNP preparations by phenolchloroform extraction, gel-purified them further, extracted them again by phenol-chloroform extraction and resuspended them in PBS. We resuspended T7 polymerase-transcribed $m_{7} \mathrm{G}$-capped $\mathrm{U} 1$ and $\mathrm{U} 2$ snRNAs in PBS. We injected 300 pg protein (50 pg RNA equivalent) of the total snRNP preparation in Danieu's buffer per embryo. We used a final concentration of 300 units per $\mathrm{ml}$ S7 micrococcal nuclease (Roche) to deplete the snRNP preparations of snRNAs $\left(30 \mathrm{~min}, 37^{\circ} \mathrm{C}\right.$ ). We quenched the reaction with EGTA at a final concentration of $2 \mathrm{mM}^{52}$. For microinjection, we adjusted the EGTA concentration in the S7-untreated sample accordingly.

Western blot. We resuspended dechorionated and deyolked embryos in cold SDS sample buffer ${ }^{47,53}$. We determined protein concentration by the Amido Schwartz Protein Assay ${ }^{54}$. For Western analysis, we analyzed $40 \mu \mathrm{g}$ of total protein extract with the following antibodies: anti-GAPDH (Novus Biologicals) at 1:5,000, anti-zebrafish coilin ${ }^{30}$ (9EA2) at $\sim 1.5 \mu \mathrm{g} \mathrm{ml}^{-1}, \mathrm{CB} 7$ (anti-U1-70K), a gift from D.L. Black (Univ. California, Los Angeles,) at 1:3, H-300 (anti-human coilin; Santa Cruz) at 1:500, anti-Sm (Y12) at 1:3, and horseradish peroxidase (HRP)coupled anti-rabbit IgG, (1:10,000, Amersham) or HRP-coupled anti-mouse IgG (1:80,000, Sigma) as secondary antibody.

Indirect immunofluorescence. We fixed embryos at room temperature $23^{\circ} \mathrm{C}$ in $4 \%(\mathrm{w} / \mathrm{v})$ paraformaldehyde (100 mM PIPES, pH 6.9, $2 \mathrm{mM} \mathrm{MgCl}_{2}, 1.25$ mM EGTA) for 15-30 min, permeabilized them for 15-30 $\mathrm{min}$ at room temperature with $0.2 \%(\mathrm{v} / \mathrm{v})$ Triton X-100, blocked them in 5\% (v/v) Normal
Goat Serum (Jackson ImmunoResearch Laboratories) and incubated them in a humidified chamber for $1 \mathrm{~h}$ at room temperature with primary antibody in PBS plus 3\% (w/v) BSA, $0.2 \%(\mathrm{w} / \mathrm{v}) \beta$-glycerophosphate and $10 \mathrm{mM} \mathrm{MgCl}_{2}$. We used secondary antibody at 1:200 dilution in the dark. We washed the samples with PBS with $10 \mathrm{mM} \mathrm{MgCl}_{2}$, rinsed them with water and mounted them on glass slides.

Antibodies for immunofluorescence. We used the following antibodies: antizebrafish coilin (9EA2, raised against a bacterially expressed fragment of zebrafish coilin) $)^{30}$ at 1:2,000, anti-Sm (Y12) at 1:1 (ref. 55), anti-TMG (K121) at 1:300 (ref. 56)(Calbiochem) and anti-SMN (1F1) at 1:100 (ref. 57). Secondary antibodies were anti-mouse conjugated with FITC and/or anti-rabbit conjugated with TRITC (Jackson ImmunoResearch Laboratories).

Microscopy. We mounted live specimens in 2-3\% (w/v) methyl cellulose in $\mathrm{E} 3$ medium $^{47}$ and fixed them in $80 \%(\mathrm{v} / \mathrm{v})$ glycerol (spectrophotometric grade, Fischer Scientific) with $25 \mathrm{mg} \mathrm{ml}^{-1}$ 1,4-diazabicyclo[2.2.2] octane (Sigma) and $2 \mu \mathrm{g} \mathrm{ml}^{-1}$ Hoechst 33342 (Sigma). We performed imaging with an Olympus FluoView 1000 confocal microscope, using UPlanApo $10 \times / \mathrm{NA}=0.4$, PlanApo $60 \times / \mathrm{NA}=1.4$ oil, and UPlanApo $60 \times / \mathrm{NA}=1.3$ oil objectives. We performed general morphological observations of embryos with Olympus SZX-12 stereomicroscope coupled to a CCD camera. We processed images using ImageJ, Bitplane Imaris, Adobe Photoshop and Illustrator (see Supplementary Methods).

Metabolic labeling. We injected into fertilized zebrafish embryos $1 \mathrm{nl}$ of $\left[\alpha_{-}{ }^{32} \mathrm{P}\right] \mathrm{UTP}\left(6,000 \mathrm{Ci} \mathrm{mmol}^{-1} ; 10 \mathrm{mCi} \mathrm{ml}^{-1}\right.$; Hartmann Analytic) followed by 1 pmol of CoilMO or CtrlMO. We left the embryos to develop at $28^{\circ} \mathrm{C}$, dechorionated them, deyolked them and subjected them to anti-Sm (Y12) immunoprecipitation as previously described ${ }^{14}$. We resolved Isolated ${ }^{32} \mathrm{P}$-labeled snRNAs on a $10 \%(\mathrm{w} / \mathrm{v})$ acrylamide/8 $\mathrm{M}$ urea gel and subjected them to phosphoimager analysis.

Quantification of spliced mRNA levels by RT-PCR. We isolated total RNA from 5- to 6-somite embryos using TRIzol Reagent (Invitrogen) and treated RNA with DNA-free kit (Ambion), using 800 ng to generate cDNA with Oligo-dT18, 5Srev (5'-AGCTTACAGCACCTGGTATTCC-3') primers and SuperScript III Reverse Transcriptase (Invitrogen). We performed quantitative PCR on a Stratagene MX3000 using the SYBR Green method (ABsolute QPCR SYBR Green ROX Mix, AB Gene) with the following PCR profile: 1 cycle of $15 \mathrm{~min}$ at $95^{\circ} \mathrm{C}$ followed by 40 cycles of $30 \mathrm{~s}$ at $95^{\circ} \mathrm{C}, 1 \mathrm{~min}$ at $60^{\circ} \mathrm{C}$ and $30 \mathrm{~s}$ at $72^{\circ} \mathrm{C}$. Primer sequences can be provided on request.

47. Westerfield, M. The Zebrafish Book: A Guide for the Laboratory Use of Zebrafish (Danio rerio) 4th edn. (University of Oregon Press, Eugene, Oregon, USA, 1995).

48. Wersig, C. \& Bindereif, A. Conserved domains of human U4 snRNA required for snRNP and spliceosome assembly. Nucleic Acids Res. 18, 6223-6229 (1990).

49. Nüsslein-Volhard, C. \& Dahm, R. eds. Zebrafish: A Practical Approach 1st edn. (Oxford University Press, Oxford, 2002).

50. Dybkov, 0 . et al. U2 snRNA-protein contacts in purified human $17 \mathrm{~S}$ U2 snRNPs and in spliceosomal A and B complexes. Mol. Cell. Biol. 26, 2803-2816 (2006).

51. Golas, M.M., Sander, B., Will, C.L., Luhrmann, R. \& Stark, H. Molecular architecture of the multiprotein splicing factor SF3b. Science 300, 980-984 (2003).

52. Krainer, A.R. \& Maniatis, T. Multiple factors including the small nuclear ribonucleoproteins $\mathrm{U} 1$ and $\mathrm{U} 2$ are necessary for pre-mRNA splicing in vitro. Cell 42, 725-736 (1985).

53. Link, V., Shevchenko, A. \& Heisenberg, C.P. Proteomics of early zebrafish embryos. BMC Dev. Biol. 6, 1 (2006).

54. Schaffner, W. \& Weissmann, C. A rapid, sensitive, and specific method for the determination of protein in dilute solution. Anal. Biochem. 56, 502-514 (1973).

55. Lerner, M.R. \& Steitz, J.A. Antibodies to small nuclear RNAs complexed with proteins are produced by patients with systemic lupus erythematosus. Proc. Natl. Acad. Sci. USA 76, 5495-5499 (1979).

56. Kramer, A., Keller, W., Appel, B. \& Luhrmann, R. The $5^{\prime}$ terminus of the RNA moiety of $U 1$ small nuclear ribonucleoprotein particles is required for the splicing of messenger RNA precursors. Cell 38, 299-307 (1984).

57. Young, P.J. et al. The exon $2 \mathrm{~b}$ region of the spinal muscular atrophy protein, SMN, is involved in self-association and SIP1 binding. Hum. Mol. Genet. 9, 2869-2877 (2000) 